\title{
A comparative study of wavelet families for electromyography signal classification based on discrete wavelet transform
}

\author{
Abdelouahad Achmamad, Atman Jbari \\ Electronic systems sensors and nano-biotechnologies, ENSET, Mohammed V University in Rabat, Morocco
}

\begin{tabular}{l} 
Article Info \\
\hline Article history: \\
Received Nov 13, 2019 \\
Revised Mar 7, 2020 \\
Accepted Mar 23, 2020 \\
\hline
\end{tabular}

\section{Keywords:}

Amyotrophic lateral sclerosis

Classification

Discrete wavelet transform

Electromyography

Support vector machine

\begin{abstract}
Automatic detection of neuromuscular disorders performed using electromyography (EMG) has become an interesting domain for many researchers. In this paper, we present an approach to evaluate and classify the non-stationary EMG signals based on discrete wavelet transform (DWT). Most often researches did not consider the effect of DWT factors on the performance of EMG signals classification. This problem is still an interesting unsolved challenge. However, the selection of appropriate mother wavelet and related level decomposition is an essential issue that should be addressed in DWT-based EMG signals classification. The proposed method consists of decomposing a raw EMG signal into different sub-bands. Several statistical features were extracted from each sub-band and six wavelet families were investigated. The feature vector was used as inputs to support vector machine (SVM) classifier for the diagnosis of neuromuscular disorders. The obtained results achieve satisfactory performances with optimal DWT factors using 10 -fold cross-validation. From the classification performances, it was found that sym14 is the most suitable mother wavelet at the 8th optimal wavelet level of decomposition. These simulation results demonstrated that the proposed method is very reliable for reducing cost computational time of automated neuromuscular disorders system and removing the redundancy information.
\end{abstract}

This is an open access article under the CC BY-SA license.

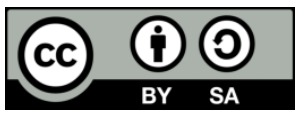

\section{Corresponding Author:}

Abdelouahad Achmamad,

Electronic Systems, Sensors and Nanobiotechnologies, ENSET,

Mohammed V University in Rabat,

Avenue des Nations Unies, Rabat, Morocco.

Email: abdelouahad.achmamad@um5s.net.ma

\section{INTRODUCTION}

Amyotrophic lateral sclerosis (ALS) is a fatal neurological disease and rapidly progressive disorder that directly attacks the central nervous system (CNS) and other neuronal cells which are responsible for controlling voluntary muscle. The onset of the ALS disease characterized by degeneration of both lower motor neurons (LMN) and upper motor neurons (UMN), with resultant muscle atrophy, muscle weakness, and damages the nerve cells of the brain and spinal cord [1-4]. The most often affected people by ALS disease are between the ages of 40 and 60, although can occur at some younger and older individuals [1, 5]. The last statistic estimate approximately five persons of every 100000 deaths each day, mostly as a result of the progress of ALS disease $[1,5]$. Hence, it would be of prime necessity to recognize early the patients affected by ALS disease for medical diagnosis.

There are various well-established clinical examinations methods for neuromuscular disorders, the frequently used are genetic and electromyography (EMG) tests, which can practically help in an early diagnostic of ALS disease $[6,7]$. In this context, electromyography has been considered to be the best clinical 
standard tool in the diagnostic of muscle disease and is commonly used for skeletal muscle investigations [8]. In fact, EMG has been traditionally utilized to assess the electrical potentials produced during contraction and relaxation of skeletal muscle [6], this technique found a wide application in multiple domains including rehabilitation, medicine prosthesis control, and clinical diagnosis [9]. In order to detect abnormalities for neuromuscular, EMG signal carries pertinent information regarding the state of the muscle. In general, the amplitude of the EMG signal varies approximately from $10 \mu \mathrm{V}$ to $5 \mathrm{mV}$ and the frequency content is concentrated in the range of $5 \mathrm{~Hz}$ to $500 \mathrm{~Hz}$ [9-11]. The EMG signal has been assumed as a non -stationary time series in which the statistical features and frequency content of the signal alter over time [12]. Due to the non-stationary characteristic of the EMG signal, it has been found that Fourier transform analysis is not suitable for EMG signal analysis. To overcome this limitation, several advanced signal processing tools have been proposed namely-time-frequency analysis, quadratic time-frequency transform, and timescale analysis approach [13]. These techniques have worked well for localization both in time and frequency [14].

The interest in the automatic identification of ALS disease has been receiving growing attention from many researchers in the last years $[15,16]$. The previous works have contributed to improving the classification accuracy of diagnostic ALS disease, however, few studies have adopted discrete wavelet transform, Abdulhamit Subasi et al employed DWT to extract statistical features from each sub-band and then feed support vector machine (SVM) classifier for EMG signals classification. Ercan Gokgoz et al. carried out a study for the diagnosis of neuromuscular disorders by the combination of DWT and decision tree algorithms [4]. A. Doulah et al., have proposed a novel idea based on the extraction of DWT coefficients-related features from dominant motor unit action potential (MUAPs) of EMG signal [17].

This study presents an automatic identification system for detecting ALS disease using the DWT technique combined with the SVM classifier. The methodology, which is proposed in this paper is shown in Figure 1. As seen in the flowchart, the proposed method involves three major steps: discrete wavelet transform application, feature extraction, and classification. When applying discrete wavelet DWT, two input factors are required: the selection of the mother wavelet and the wavelet level of decomposition. It is important to highlight that our work focuses mainly on the influence of DWT related factors on the performance of classification results of neuromuscular disorders system. The DWT is first applied to decompose EMG signals into a specific frequency band and then DWT coefficient features are extracted from each band and fed into support vector machines SVMs algorithm for EMG signals classification. The remainder of this paper is arranged as follows. Section 2 provides an overview of the experimental data and a brief description of the basic background theory of DWT, while the classification model and feature extraction are explicated in section 3. The performance results and comparison with related literature studies are presented in section 4 . Finally, the conclusion of this work is given in the last section.

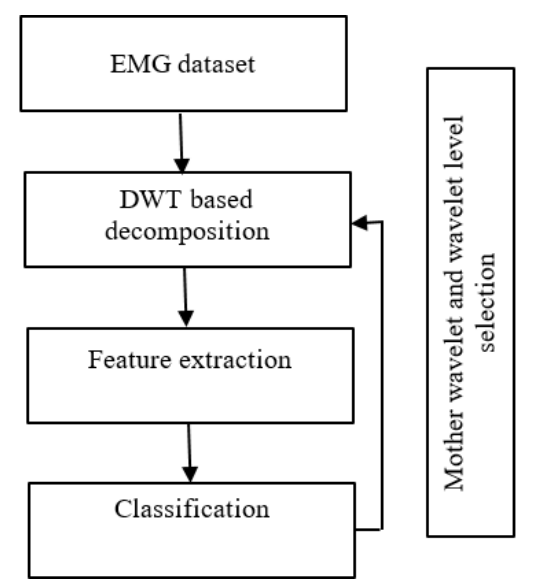

Figure 1. Block diagram for the wavelet-based automated classification system

\section{MATERIALS AND METHODS}

\subsection{Data acquisition}

The data set in this work was taken from publicly available at Clinical Neurophysiology, Rigs hospital, Copenhagen, Denmark. The EMG signals were recorded by inserting a fine wire electrode inside the biceps brachii (long head\& court head) from five places in the muscle at three levels of insertion (deep, medium, low). A standard concentric needle electrode offers better selectivity of motor unit action potentials with a low spatial gradient [7]. The complete data consists of two classes, amyotrophic lateral 
sclerosis ALS patients' class, and normal class. ALS class contains eight control subjects; four females and four males aged 35-67 years old, whereas the normal class contains ten subjects aged 21-37 years, four females and six males were in good physical shape and have any history of neuromuscular disorders before. The EMG signals are sampled at a $20 \mathrm{kHz}$ and digitalized at16-bit A/D resolution. The high and low pass filters of the EMG amplifier were set at $2 \mathrm{~Hz}$ and $10 \mathrm{kHz}$ respectively [3, 4, 18]. A typical example of ALS and normal EMG signal which are selected from data sets are shown in Figure 2.

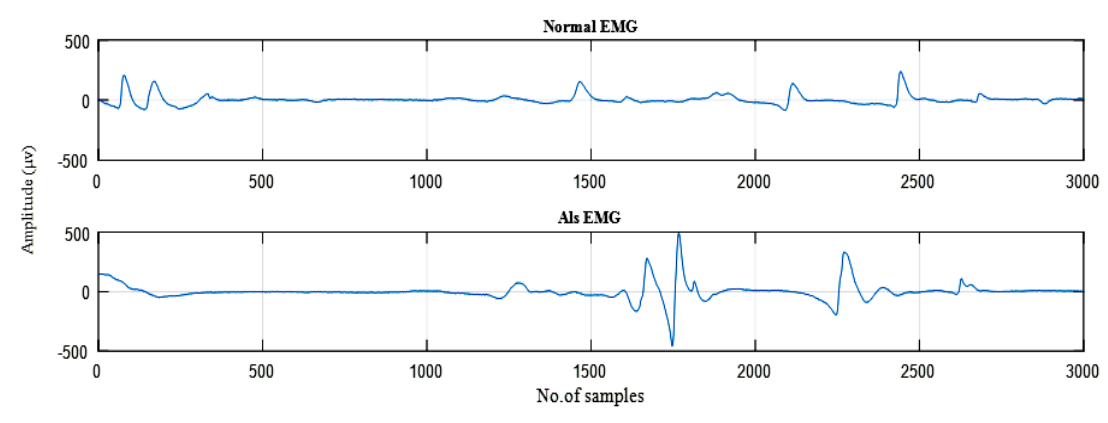

Figure 2. EMG data pattern taken from different subjects: ALS EMG signal (lower) and normal EMG signal (upper) for 3000 sample number

\subsection{EMG signal decomposition based on DWT}

Over the past three decades, continuous wavelet transform (CWT) has become more popular because of its particularly interesting properties. In fact, CWT is a multi-scale resolution tool and is suitable for analyzing non-stationary signal like the EMG signal better than any other existing transforms [18]. Continuous wavelet transform was developed to replace the classical short-time Fourier transform (STFT), except that, it has solved some of STFT drawbacks which are resolution and the inherent weaknesses of the fixed window size $[19,20]$. According to the principle of CWT, the analyzed signal is convolved with mother wavelet function $\psi(\mathrm{t})$. Hence, the CWT of the EMG signal provided by the following $[19,21]$ :

$$
C W T_{x}(u, s)=\frac{1}{\sqrt{s}} \int_{-\infty}^{+\infty} \operatorname{emg}(t) \psi_{u, s}^{*}(t) d t
$$

where

$$
\psi_{\mathrm{u}, \mathrm{s}}(\mathrm{t})=\frac{1}{\sqrt{s}} \psi\left(\frac{t-u}{s}\right)
$$

In (1) and (2), $\mathrm{s}$ and $\mathrm{u}$ are real numbers $\mathrm{s} \neq 0$, as well as are used as scaling and shifting parameters respectively.

Discrete wavelet transform DWT is a more powerful time-frequency/time scale representation. Moreover, it offers an easy advantage to implement and low computation cost than other classical methods like discrete Fourier transform (DFT) and discrete cosine transform (DCT) [18, 22]. Discrete wavelet transform is obtained by discretizing the scaling $\mathrm{s}$ and shifting $\mathrm{u}$ parameters: $s=s_{0}{ }^{j}$ and $u=\mathrm{k} s_{0}{ }^{\mathrm{j}}$, with $k, j \in \mathbb{Z}$. By substituting in (2) DWT could be written as:

$$
\operatorname{DWT}(\mathrm{j}, \mathrm{k})=\sum_{\mathrm{n}=0}^{\mathrm{N}-1} \operatorname{emg}(n) \cdot \frac{1}{\sqrt{s_{0}}{ }^{j}} \psi^{*}\left(\frac{\mathrm{n}-\mathrm{k} s_{0}{ }^{j}}{s_{0}{ }^{j}}\right)
$$

For a dyadic DWT: $s_{0}=2$.

where the superscript $*$ denotes a complex conjugate, $N$ the length of $E M G$ signal and $j=1,2, \ldots M$ are the wavelet decomposition levels.

The multiresolution decomposition algorithm was originally proposed by S. Mallat in 1989 The algorithm splits the initial analyzed signal into two components known as detail coefficients $c D s$ and approximation coefficients $c A s$ [23]. These two specific components are efficiently generated by means of a pair of high pass and low pass wavelet filters. After each decomposition, the down sampling frequency of signals is done to avoid generating redundant data. This procedure is given by the following expression [24]:

$$
\left\{\begin{array}{c}
d_{j}(n)=\sum_{k} \operatorname{HiD}(2 n-k) a_{j-1}(k) \\
a_{j}(n)=\sum_{k} \operatorname{LoD}(2 n-k) a_{j-1}(k) \quad \mathrm{j} \geq 1 \\
a_{0}(n)=\operatorname{emg}(n)
\end{array}\right.
$$


where emg(n) is the analyzed $E M G$ signal. $n=1,2, \ldots N, N$ is the length of $E M G$ signal. The low pass filter is denoted by $\operatorname{LoD}(n)$ while the high pass filter is denoted by $\operatorname{HiD}(n)$. At each level $j$, the high pass filter produces detail coefficients information $c D s$, while the low pass filter associated with scaling function produces coarse approximations coefficients $c A s$. The detail and approximation coefficients of ALS and normal EMG signals for 3000 samples are shown in Figure 3.

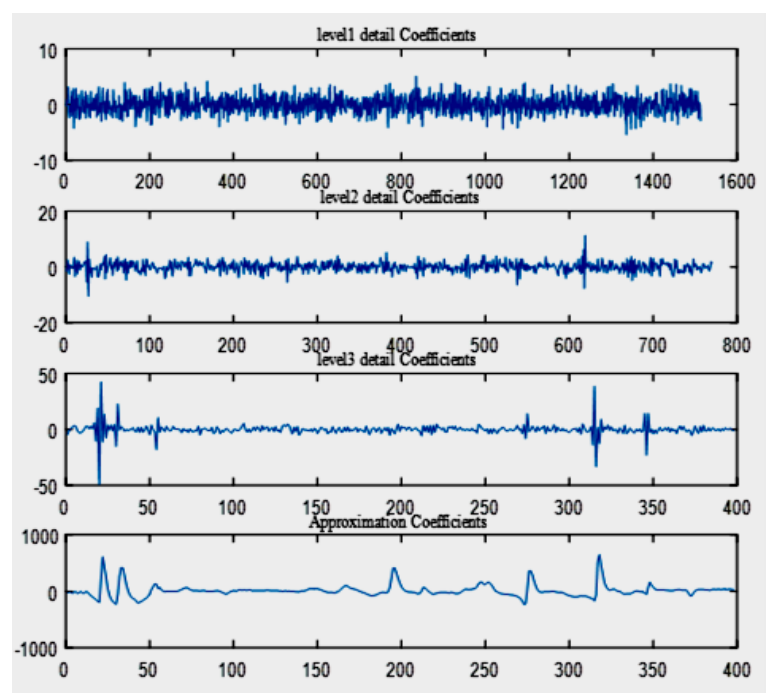

(a)

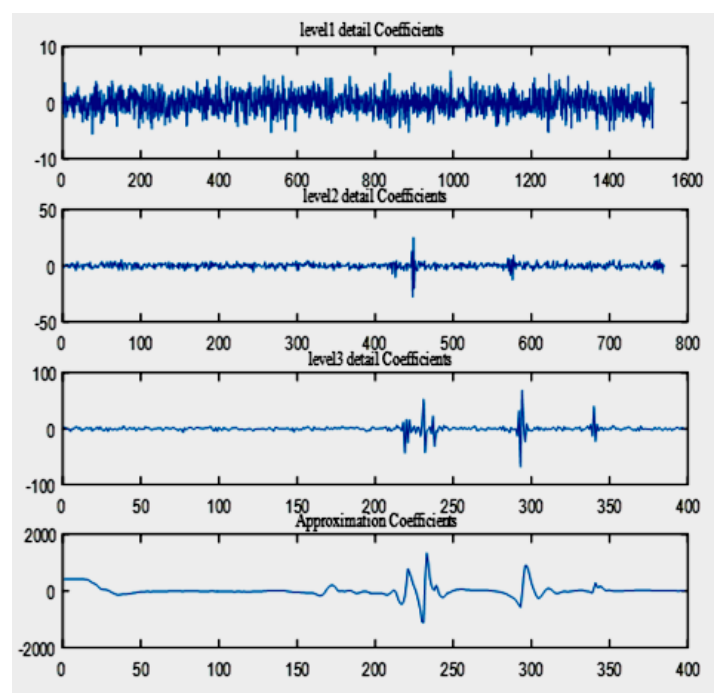

(b)

Figure 3. Discrete wavelet coefficients for symlet 14, (a) DWT coefficients of normal EMG signal, (b) DWT coefficients of ALS EMG signal

\subsection{Frequency band}

In each wavelet level of decomposition, the frequency band corresponding to detail and approximation coefficients are given as follow [25]:

$$
d_{j}(n):\left[\frac{f_{s}}{2^{j+1}}, \frac{f_{s}}{2^{j}}\right], \quad a_{j}(n):\left[0, \frac{f_{s}}{2^{j+1}}\right]
$$

where $f_{s}$ is the sampling rate of the EMG signal.

As given in our dataset, the sampling frequency is $20 \mathrm{kHz}$. Hence, the frequency range of each sub-band after DWT is depicted as shown in Figure 4.

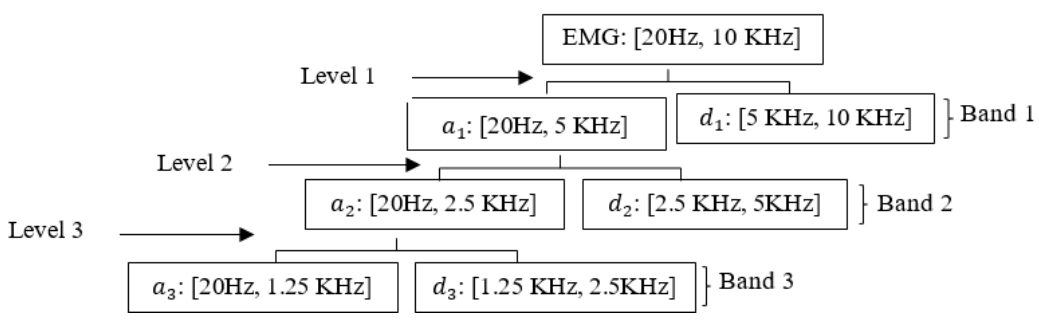

Figure 4. Structure of frequency band for third level decomposition under a $20 \mathrm{KHz}$ sampling rate

\subsection{Level of decomposition selection}

The level of decomposition plays an essential role in the optimization of the computation time of the neuromuscular disorders detection system. In order to avoid time waste, the level of decomposition corresponding to the performance of the classification must be carefully chosen. In this present work, we have tested the classification performance based on choosing the level from 1 to Maxlev [26].

$$
\text { Maxlev }=\text { fix }\left[\log _{2}\left(\frac{\mathrm{N}}{(\mathrm{Lw}-1)}\right)\right]
$$

where $\mathrm{N}$ is the length of EMG signal and Lw is the length of the mother wavelet of high-pass and low-pass filters, fix is round operator toward zero. 


\subsection{Wavelet selection}

Wavelet selection will be carried out based on several wavelets analysis tests. However, we must also consider the wavelet properties such as vanishing moment, compact support, orthogonality, and symmetry in wavelet selecting. The analyzing wavelets used are presented in the following Table 1 .

Table 1. wavelet family with its properties

\begin{tabular}{|c|c|c|c|c|c|c|}
\hline $\begin{array}{l}\text { Wavelet } \\
\text { family }\end{array}$ & Wavelet Member & Orthogonal & Compact support & Filters length & Support width & $\begin{array}{l}\text { Vanishing } \\
\text { moment }\end{array}$ \\
\hline $\mathrm{Db}$ & 'db1', 'd45' & Yes & Yes & $2 N$ & $2 N-1$ & $N$ \\
\hline Coif & 'coifl', 'coif5' & Yes & Yes & $6 N$ & $6 N-1$ & $2 N$ \\
\hline Sym & 'sym1,'sym45', & Yes & Yes & $2 N$ & $2 N-1$ & $N$ \\
\hline Bior & $\operatorname{biorNd}, \mathrm{Nr}$ & Yes & Yes & $\max (2 N r, 2 N d)+2$ & $\begin{array}{l}2 N+1 \text { for rec. } \\
2 N d+1 \text { for dec. }\end{array}$ & $N r$ \\
\hline Rbio & $\operatorname{rbiorNd,Nr}$ & No & Yes & $\max (2 N d, 2 N r)+2$ & $\begin{array}{l}2 N+1 \text { for rec. } \\
2 N r+1 \text { for dec. }\end{array}$ & $N r$ \\
\hline dmey & 'dmey' & - & - & - & - & - \\
\hline
\end{tabular}

Where $\mathrm{Nr}, \mathrm{Nd}$, and $\mathrm{N}$ are the orders: $\mathrm{r}$ for reconstruction and $\mathrm{d}$ for decomposition.

\section{CLASSIFICATION METHOD AND FEATURES EXTRACTION}

In this study, a support vector machine (SVM) was used as the classifier model. It is one of the most commonly used in the EMG signals classification, as it is a simple and accurate method for the identification of neuromuscular disorders.

\subsection{Support vector machine SVM}

Support vector machine classifier is a type of supervised machine learning algorithm, it has a particular advantage in solving non-linear separation and high dimensional features space problem. A classification task basically involves training and testing data that contain data instances, each instance consists of two attribute features and class label. Supervised training used to build a model that can predict the class label of the data testing. The main objective of SVM is to separate the data between two classes by finding the optimal separating hyperplane with the widest margin. The distance between support vectors and hyperplane must be as far as possible [27-29]. Given a training set of instances label pairs $\left\{x_{i}, y_{i}\right\}, x_{i} \in \mathbb{R}^{d}$, $\mathrm{d}$ is dimensional instances in the input space, and the associated $y_{i} \in\{-1,+1\}$ represents a class label. The mathematical equation of a hyperplane is:

$$
f(x)=w^{T} x+b=0
$$

supposing that one data set is nonlinear, we need to create the SVM regression function:

$$
\mathrm{f}(\mathrm{x})=\mathrm{w}^{\mathrm{T}} \varphi(\mathrm{x})+\mathrm{b}
$$

where $w \in \mathbb{R}^{d}$ is the weight vector, $\mathrm{b}$ is the bias term and is $\varphi(x)$ is the nonlinear function.

SVM classifier requires to find solution of the following quadratic optimization problem [28]:

$$
\min _{w, b, \xi}\left(\frac{1}{2} w^{T} w+C \sum_{i=1}^{N} \xi_{i}\right)
$$

subject to $\quad y_{i}\left(f\left(x_{i}\right)\right) \geq 1-\xi_{i}, i=1,2, \ldots, N$

$$
\xi_{i} \geq 0, C>0
$$

where $C$ is the penalty parameter of the error term, $\xi_{i}$ is a flexible variable and $\mathrm{N}$ is the number of data.

In practical application, the binary classification problems do not have a simple hyperplane as a useful separating criterion. To overcome this problem, the SVM classifier uses several kernel functions [30]. In his paper, the classification model is built by using radial basis function (RBF) which can be written as follow:

$$
\mathrm{K}\left(x_{i}, x_{j}\right)=\varphi(x)^{T} \varphi\left(x_{i}\right)=e^{\frac{-\left\|\mathrm{x}-x_{i}\right\|^{2}}{2 \sigma^{2}}}, \sigma \neq 0
$$

\subsection{Feature extraction based on discrete wavelet transform DWT}

The DWT has been used in many practical applications such as noise cancellation and feature extraction. In this study, the proposed feature extraction has a significant effect on performance 
classification. Therefore, it is important to select the optimal feature with useful information, because the performance result degrades when one or more features are irrelevant. Subsequently, various features of wavelet coefficients are computed by using DWT, the features vectors obtained will be used in the training and classification phase. The expressions of coefficients-related features are presented in the following Table $2[19,31]$ :

Table 2. Features and their expressions

\begin{tabular}{|c|c|c|}
\hline Feature Extraction & Mathematical expression & Definition \\
\hline Root mean square value & $\operatorname{RMS}_{c_{j}}=\left.\left|\frac{1}{\mathrm{n}} \sum_{\mathrm{i}=1}^{\mathrm{n}}\right| c_{\mathrm{ji}}\right|^{2}$ & $\begin{array}{l}\text { Measure the root mean square value of } \\
\text { the wavelet coefficients in each sub-band. }\end{array}$ \\
\hline $\begin{array}{l}\text { Normalized root mean } \\
\text { square }\end{array}$ & $\operatorname{nRMS}_{c_{j}}=1-\frac{\operatorname{RMS}_{c_{j}}}{\left|\max \left(c_{\mathrm{ji}}\right)\right|}$ & $\begin{array}{l}\text { It operates on the basis of calculating RMS of } \\
\text { the wavelet coefficients in each sub-band with amplitude } \\
\text { normalized by wavelet coefficients peak value. }\end{array}$ \\
\hline Energy & $\mathrm{E}_{c_{j}}=\sum^{\mathrm{n}}\left|\mathrm{c}_{\mathrm{ji}}\right|^{2}$ & $\begin{array}{l}\text { Measure the energy value of the wavelet coefficients in } \\
\text { each sub-band. }\end{array}$ \\
\hline Normalized energy & $n E_{c_{j}}=\frac{\mathrm{E}_{c_{j}}}{B_{j i}}$ & $\begin{array}{l}\text { Measure the ratio of energy value of } \\
\text { the size sub-band. }\end{array}$ \\
\hline $\begin{array}{l}\text { Maximal coefficient } \\
\text { Minimal coefficient }\end{array}$ & $\begin{array}{ll}\max \left(c_{\mathrm{ji}}\right) & (15) \\
\min \left(c_{\mathrm{ji}}\right) & (16)\end{array}$ & $\begin{array}{l}\text { Maximum value of the coefficients in each sub-band. } \\
\text { Minimum value of the coefficients in each sub-band. }\end{array}$ \\
\hline Crest factor value & $C F_{c_{j}}=\frac{\left|\max \left(c_{\mathrm{ji}}\right)\right|}{\operatorname{RMS}_{c_{j}}}$ & $\begin{array}{l}\text { Defined as the peak value of } c_{\mathrm{ji}} \text { divided by } \\
\text { the RMS of the coefficients in each sub-band. }\end{array}$ \\
\hline Kurtosis & Kurtosis $_{c_{j}}=\frac{\frac{\sum_{i}^{n}\left(c_{j i}-\operatorname{Mean}_{c_{j}}\right)^{4}}{n}}{S T D^{4}}$ & $\begin{array}{l}\text { Measure of tailedness of the coefficients in each } \\
\text { sub-band. }\end{array}$ \\
\hline Skewness & Skewness $_{c_{j}}=\frac{\frac{\sum_{i=1}^{n}\left(c_{j i}-\operatorname{Mean}_{c_{j}}\right)^{3}}{n}}{\operatorname{STD}_{c_{j}}{ }^{3}}$ & $\begin{array}{l}\text { Measure the lack of symmetry of the coefficients in each sub- } \\
\text { band. }\end{array}$ \\
\hline Variance & $\operatorname{Var}_{c_{j}}=\frac{1}{n-1} \sum_{i=1}^{n}\left|c_{j i}-\operatorname{Mean}_{c_{j}}\right|^{2}$ & $\begin{array}{l}\text { Measure of how far a set of numbers is spread out from } \\
\text { mean. }\end{array}$ \\
\hline Standard deviation & $\operatorname{STD}_{c_{j}}=\sqrt{\frac{\sum_{\mathrm{i}=1}^{\mathrm{n}}\left(\mathrm{c}_{\mathrm{ij}}-\mathrm{Mean}_{c_{j}}\right)^{2}}{\mathrm{n}}}$ & $\begin{array}{l}\text { A measure of dispersion of the coefficients in each } \\
\text { sub-band. }\end{array}$ \\
\hline $\begin{array}{l}\text { Normalized standard } \\
\text { deviation }\end{array}$ & $\mathrm{nSTD}_{c_{j}}=\frac{\mathrm{STD}_{c_{j}}}{\max \left(\mathrm{c}_{\mathrm{j}}\right)-\min \left(\mathrm{c}_{\mathrm{j}}\right)}$ & $\begin{array}{l}\text { Measure ratio standard deviation of subtraction } \\
\text { of maximum and minimum values in each sub-band. }\end{array}$ \\
\hline Median value & $\begin{array}{l}\operatorname{Mean}_{c_{j}}=\frac{\sum_{\mathrm{i}=1}^{\mathrm{n}} \mathrm{c}_{\mathrm{ij}}}{\mathrm{n}} \\
\left\{\begin{array}{c}M d_{c_{j}}=\frac{1}{2}\left(c_{\frac{n}{2}}+c_{\frac{n}{2}}\right), n \text { is even } \\
M d_{c_{j}}=\frac{c_{(n-1)}}{2}, n \text { is odd }\end{array}\right.\end{array}$ & $\begin{array}{l}\text { Represents the average value absolute value of the } \\
\text { coefficients in each sub-band. } \\
\text { A sample value separating higher from the lower half } \\
\text { of wavelet coefficients. }\end{array}$ \\
\hline Zero crossing & $\begin{array}{c}\mathrm{ZC}_{c_{j}} \sum_{\mathrm{i}=1}^{\mathrm{n}}\left[\begin{array}{c}\left.\operatorname{sgn}\left(\mathrm{c}_{\mathrm{ji}} * \mathrm{c}_{\mathrm{ji}+1}\right) \cap\left|c_{\mathrm{ji}}-\mathrm{c}_{\mathrm{ji}+1}\right|\right] \\
\geq \text { threshold }\end{array}\right] \\
\operatorname{sgn}=\left\{\begin{array}{c}1, \text { if } \mathrm{c} \geq \text { threshold } \\
0, \text { otherwise }\end{array}\right.\end{array}$ & $\begin{array}{l}\text { It is a number of times that amplitude values of } \\
\text { the wavelet coefficients cross zero amplitude level. } \\
\text { To avoid low voltage fluctuations, the threshold } \\
\text { condition is applied. }\end{array}$ \\
\hline
\end{tabular}

From the features expression, $i=1,2, \ldots n, \mathrm{n}$ is the length of each band and $j=1,2, \ldots$ Maxlev are the wavelet decomposition levels while $\mathrm{c}_{\mathrm{ji}}$ denoted of detail coefficients from each level of decomposition and approximation coefficients at last decomposition level.

\section{RESULTS AND DISCUSSION}

The purpose of this work is to study the EMG signals acquired from the normal and affected brachial biceps by applying DWT in order to determine the suitable mother wavelet and level of decomposition, which yield the best classification performances. The EMG signals are decomposed into maximum level decomposing using different mother wavelets, fifteen coefficients-related features are extracted and then passed through the SVM classifier with the RBF kernel for EMG signals classification. To further analyze the robust performance of the neuromuscular diagnostic system, one of the well-known crossvalidation $(\mathrm{CV})$ algorithms named 10 -fold $\mathrm{CV}$ is deployed as data set partitioning with a total of 18 subjects.

Cross-validation is a measurement for evaluating the performance of predictive model and ensuring the reliable performance of SVM classifier. There are many types of cross validation such as k-fold, hold out, and leave one cross-validation, we picked up k-fold cross validation for assessment. K-fold cross validation 
$(\mathrm{k}-\mathrm{CV})$ is a method of broken the original data randomly into the $k$ subsets for machine learning technique routine SVM with $\mathrm{k}-\mathrm{CV}$ (k-CV SVM), then $k-1$ subsets are used as training data and the single subset data remaining is regarded as data test of classification model. The whole process is repeated k-folds time and each $\mathrm{k}$ subset is employed exactly once as data testing while the $k-1$ subsets are employed as training data before data testing. The performance of classification is calculated for each data testing, then the final average of the performance is obtained by averaging the performances of all data testing. SVM classifier is performed by using three parameters in order to examine our neuromuscular disorders system. The three parameters are accuracy, sensitivity and specificity with their mathematical expressions written as follows:

$$
\begin{aligned}
& \text { Accuracy }(\%)=\frac{\mathrm{TP}+\mathrm{TN}}{\mathrm{TP}+\mathrm{TN}+\mathrm{FN}+\mathrm{FP}} \times 100 \% \\
& \text { Sensitivity }(\%)=\frac{\mathrm{TP}}{\mathrm{TP}+\mathrm{FN}} \times 100 \% \\
& \text { Specificity }(\%)=\frac{\mathrm{TN}}{\mathrm{TN}+\mathrm{FP}} \times 100 \%
\end{aligned}
$$

TP, TN, FP and FN are measured from matrix confusion which contains information about prediction and actual labels [32]. Where:

TP : true positives (is the number of normal EMG signals identified as normal EMG signals)

TN : true negatives (is the number of ALS EMG signals classified as ALS EMG signals)

FP : false positives (is the number of ALS EMG signals recognized as normal EMG signals)

FN : false negatives (is the number of normal EMG signals distinguished as ALS EMG signals)

As mentioned in the introduction, our method consists to study the effect of different wavelet family and decomposition level on the DWT based neuromuscular diagnostic. In our proposed approach we investigate some optimization techniques to further reduce computation cost and increase the accuracy of our diagnostic system. This approach has been never used for classification of the neuromuscular disorders before. We implement our approach for different mother wavelets and related levels of decomposition. Table 3 shows

\begin{tabular}{|c|c|c|c|c|c|c|c|}
\hline $\begin{array}{l}\text { Wavelet } \\
\text { Family }\end{array}$ & $\begin{array}{l}\text { Wavelet } \\
\text { member }\end{array}$ & $\begin{array}{l}\text { Maximum } \\
\text { level }\end{array}$ & $\begin{array}{l}\text { Best average accuracy } \pm \\
\text { standard deviation /optimal } \\
\text { level }\end{array}$ & $\begin{array}{l}\text { Wavelet } \\
\text { Family }\end{array}$ & $\begin{array}{l}\text { Wavelet } \\
\text { member }\end{array}$ & $\begin{array}{l}\text { Maximum } \\
\text { level }\end{array}$ & $\begin{array}{l}\text { Best average accuracy } \pm \\
\text { standard deviation/optimal } \\
\text { level }\end{array}$ \\
\hline \multirow[t]{15}{*}{$\overline{\mathrm{Db}}$} & $\mathrm{db} 1$ & 17 & $92.01 \pm 0.51 \% / 14$ & \multirow[t]{15}{*}{ Sym } & sym1 & 17 & $92.0 \pm 0.30 \% / 14$ \\
\hline & $\mathrm{db} 2$ & 16 & $91.94 \pm 0.80 \% / 8$ & & sym2 & 16 & $91.43 \pm 0.56 \% / 8$ \\
\hline & $\mathrm{db} 3$ & 15 & $92.04 \pm 0.50 \% / 8$ & & sym3 & 16 & $92.04 \pm 0.63 \% / 8$ \\
\hline & $\mathrm{db} 4$ & 15 & $92.16 \pm 0.56 \% / 8$ & & sym4 & 15 & $92.04 \pm 0.24 \% / 8$ \\
\hline & db5 & 14 & $92.67 \pm 0.49 \% / 12$ & & sym5 & 14 & $93.70 \pm 0.63 \% / 9$ \\
\hline & $\mathrm{db} 6$ & 14 & $92.46 \pm 0.46 \% / 12$ & & sym6 & 14 & $93.45 \pm 0.29 \% / 12$ \\
\hline & $\mathrm{db} 7$ & 14 & $91.71 \pm 0.43 \% / 9$ & & sym7 & 14 & $92.19 \pm 0.73 \% / 9$ \\
\hline & $\mathrm{db} 8$ & 14 & $91.68 \pm 0.45 \% / 7$ & & sym8 & 14 & $92.94 \pm 0.59 \% / 12$ \\
\hline & db9 & 13 & $92.09 \pm 0.31 \% / 10$ & & sym9 & 13 & $92.19 \pm 0.54 \% / 8$ \\
\hline & db10 & 13 & $94.2 \pm 0.86 \% / 12$ & & sym10 & 13 & $92.21 \pm 0.46 \% / 9$ \\
\hline & db11 & 13 & $92.01 \pm 0.47 \% / 8$ & & sym11 & 13 & $93.19 \pm 0.23 \% / 9$ \\
\hline & $\mathrm{db} 12$ & 13 & $93.45 \pm 0.58 \% / 8$ & & sym 12 & 13 & $92.77 \pm 0.31 \% / 8$ \\
\hline & $\mathrm{db} 13$ & 13 & $93.70 \pm 0.34 \% / 9$ & & sym13 & 13 & $91.53 \pm 0.47 \% / 7$ \\
\hline & $\mathrm{db} 14$ & 13 & $92.16 \pm 0.66 \% / 9$ & & sym14 & 13 & $94.20 \pm 0.53 \% / 8$ \\
\hline & $\mathrm{db} 15$ & 13 & $91.53 \pm 0.75 \% / 9$ & & sym 15 & 13 & $93.07 \pm 0.57 \% / 8$ \\
\hline \multirow[t]{15}{*}{ Bior } & bior1.1 & 17 & $91.56 \pm 0.81 \% / 11$ & \multirow[t]{15}{*}{ Rbio } & rbio1.1 & 17 & $92.11 \pm 0.428 \% / 14$ \\
\hline & bior1.3 & 15 & $90.93 \pm 0.42 \% / 9$ & & rbio1.3 & 15 & $91.51 \pm 0.69 \% / 7$ \\
\hline & bior1.5 & 14 & $91.25 \pm 0.37 \% / 7$ & & rbio1.5 & 14 & $91.9 \pm 30.44 \% / 9$ \\
\hline & bior2.2 & 15 & $92.74 \pm 0.57 \% / 8$ & & rbio 2.2 & 15 & $92.11 \pm 0.60 \% / 8$ \\
\hline & bior2.4 & 14 & $92.51 \pm 0.35 \% / 8$ & & rbio2.4 & 14 & $91.18 \pm 0.35 \% / 7$ \\
\hline & bior2.6 & 14 & $92.24 \pm 0.33 \% / 8$ & & rbio2.6 & 14 & $92.34 \pm 0.29 \% / 12$ \\
\hline & bior 2.8 & 13 & $92.06 \pm 0.47 \% / 8$ & & rbio 2.8 & 13 & $92.16 \pm 0.67 \% / 8$ \\
\hline & bior3.1 & 16 & $91.36 \pm 0.59 \% / 7$ & & rbio3.1 & 16 & $91.25 \pm 0.51 \% / 8$ \\
\hline & bior3.3 & 15 & $91.28 \pm 0.44 \% / 7$ & & rbio3.3 & 15 & $91.20 \pm 0.49 \% / 8$ \\
\hline & bior3.5 & 15 & $93.95 \pm 0.60 \% / 9$ & & rbio3.5 & 14 & $91.83 \pm 0.53 \% / 9$ \\
\hline & bior3.7 & 14 & $92.44 \pm 0.23 \% / 11$ & & rbio3.7 & 14 & $92.14 \pm 0.54 \% / 10$ \\
\hline & bior3.9 & 13 & $91.76 \pm 0.23 \% / 10$ & & rbio3.9 & 13 & $92.36 \pm 0.70 \% / 9$ \\
\hline & bior4.4 & 13 & $91.73 \pm 0.28 \% / 8$ & & rbio4.4 & 13 & $91.08 \pm 0.46 \% / 6$ \\
\hline & bior5.5 & 14 & $91.98 \pm 0.57 \% / 9$ & & rbio5.5 & 13 & $91.78 \pm 0.29 \% / 8$ \\
\hline & bior6.8 & 13 & $92.74 \pm 0.19 \% / 9$ & & rbio6.8 & 13 & $92.36 \pm 0.53 \% / 9$ \\
\hline \multirow[t]{3}{*}{ Coif } & coif1 & 15 & $91.93 \pm 0.41 \% / 6$ & \multirow[t]{2}{*}{ Coif } & coif4 & 13 & $91.63 \pm 0.44 \% / 8$ \\
\hline & coif2 & 14 & $92.34 \pm 0.24 \% / 8$ & & coif5 & 13 & $91.00 \pm 0.53 \% / 6$ \\
\hline & coif3 & 13 & $91.46 \pm 0.67 \% / 9$ & Dmey & dmey & 11 & $92.31 \pm 0.43 \% / 8$ \\
\hline
\end{tabular}
the classification accuracies with optimal level obtained by our approach.

Table 3. Performance results of the proposed method

Bulletin of Electr Eng \& Inf, Vol. 9, No. 4, August 2020 : 1420 - 1429 
From Table 3, it is clear that the decomposition level affects the accuracy substantially regardless of the mother wavelets. The experiments are carried out by using MATLAB 2015a software on Windows $10 P C$ with Intel Core $i 5$ processor $2.5 \mathrm{GHz}$. As can be seen, the results proved that there is no significant difference in classification accuracy between different mother wavelets. All mother wavelets have achieved average accuracy above (91\%) at different optimal decomposition level. Based on classification accuracy, the best member wavelets selected from each family are db10, sym14, bior3.5, rbio3.9, dmey, coif2. However, the highest average accuracy(94.2\%) was achieved by Daubechies (order 10) and Symelet (order 14) at 12th decomposition level and at 8th decomposition level respectively. This satisfactory accuracy of $\mathrm{db} 10$ and sym14 justified by their good properties.

Simulation results in Figure 5 indicate that the classification performance increase with the increasing of decomposition level. Moreover, from our obtained result it was observed that there is no significant change in classification performance after optimal decomposition level of candidate mother wavelets. It is clear that more level of decomposition provides a detailed illustration of the EMG signal, but it can generate feature redundancy and noise, which lead to increased computation cost and reduced classification performance of diagnostic system.
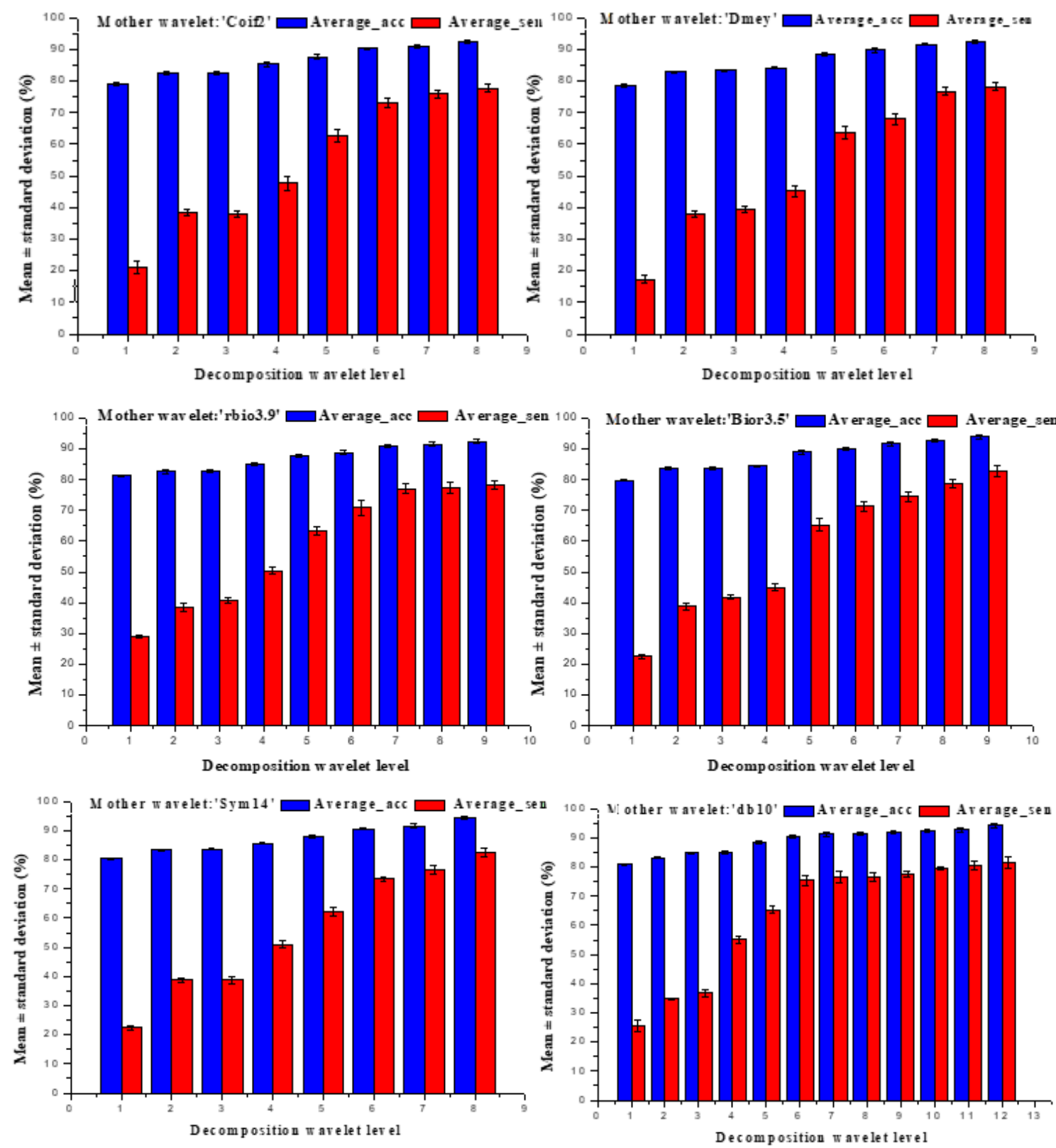

Figure 5. Classification result of the six selected wavelet member versus decomposition levels 
As a result, all the results in Tables 3, 4, and Figure 5 show the same conclusion: According to the highest performance classification the suitable mother wavelets of each wavelet family are finally selected with their optimal level of decomposition. We can conclude that the Symelt (order14) is found to be the best for classifying EMG signals which indicate high-performance classification and optimal decomposition compared to other candidate mother wavelets. The results of our approach are comparable with other similar and recent works in the neuromuscular diagnostic. Table 5 gives the classification accuracies of the previous studies and our work. As can be seen, our method achieved promising classification accuracy.

Table 4. Summary of results

\begin{tabular}{llllll}
\hline Wavelet selected & Average accuracy $(\%)$ & Average sensitivity $(\%)$ & Average specificity (\%) & Max level & Level selected \\
\hline db10 & $94.20 \pm 0.86 \%$ & $81.63 \pm 2.12 \%$ & $98.32 \pm 0.55 \%$ & 13 & 12 \\
bior3.5 & $93.95 \pm 0.60 \%$ & $82.65 \pm 1.86 \%$ & $97.65 \pm 0.45 \%$ & 15 & 9 \\
coif2 & $92.34 \pm 0.24 \%$ & $77.65 \pm 1.31 \%$ & $96.92 \pm 0.21 \%$ & 14 & 8 \\
sym14 & $\mathbf{9 4 . 2 0} \pm \mathbf{0 . 5 3} \%$ & $\mathbf{8 2 . 6 5} \pm \mathbf{1 . 5 5} \%$ & $\mathbf{9 8 . 6 6} \pm \mathbf{0 . 3 2} \%$ & $\mathbf{1 3}$ & $\mathbf{8}$ \\
dmey & $92.31 \pm 0.43 \%$ & $78.16 \pm 1.19 \%$ & $96.95 \pm 0.36 \%$ & 11 & 8 \\
rbior3.9 & $92.36 \pm 0.70 \%$ & $78.26 \pm 1.27 \%$ & $96.98 \pm 0.74 \%$ & 13 & 9 \\
\hline
\end{tabular}

Table 5. Performances comparison of EMG classification methods

\begin{tabular}{llll}
\hline Methods & Total subjects & Total classes & Highest classification accuracy (\%) \\
\hline TQWT [33] & 18 & 2 & $\mathbf{9 5 \%}$ \\
IEMD [4] & 18 & 2 & $\mathbf{9 2 . 6 6 \%}$ \\
Our approach & 18 & 2 & $\mathbf{9 4 . 2 0 \%}$ \\
\hline
\end{tabular}

\section{CONCLUSION}

The discrete wavelet transform is an efficient method for evaluating electromyography signal more than other methods. This work is concerned with the selection of optimal DWT factors for neuromuscular disorders based on performances classification. For performing the classification process, SVM classifier was proposed. The simulation results show that the proposed method can make a powerful analysis. After doing all the test of the mother wavelets, the sym14 has been selected as a suitable mother wavelet at 8th levels of decomposition for EMG signals classification. It has been found that performances classifications are sensitive to decomposition level regardless mother wavelet. Finally, this study has been done to demonstrate clearly that the selection of mother wavelet and decomposition level plays also an important role in terms of classification performance of neuromuscular disorders.

\section{REFERENCES}

[1] S. Niedermeyer, M. Murn, and P. J. Choi, "Respiratory failure in amyotrophic lateral sclerosis," Chest, vol. 155, no. 2, pp. 401-408, 2019.

[2] P. Corcia, S. Beltran, G. Lautrette, S. Bakkouche, and P. Couratier, "Staging amyotrophic lateral sclerosis: A new focus on progression," Revue Neurologique, vol. 174, no. 5, pp. 277-282, 2019.

[3] V. K. Mishra, V. Bajaj, A. Kumar, D. Sharma, and G. K. Singh, "An efficient method for analysis of EMG signals using improved empirical mode decomposition," AEU-International Journal of Electronics and Communications, vol. 72, pp. 200-209, 2017.

[4] E. Gokgoz and A. Subasi, "Biomedical signal processing and control comparison of decision tree algorithms for EMG signal classification using DWT," Biomedical Signal Processing and Control, vol. 18, pp. 138-144, 2015.

[5] B. Oskarsson, T. F. Gendron, and N. P. Staff, "Amyotrophic lateral sclerosis: An update for 2018," Mayo Clinic Proceedings, vol. 93, no. 11, pp. 1617-1628, 2018.

[6] R. M. Enoka and J. Duchateau, "Inappropriate interpretation of surface EMG signals and muscle fiber characteristics impedes understanding of the control of neuromuscular function," Journal of Applied Physiology, vol. 119 , no. 12 , pp. 1516-1518, 2015.

[7] I. M. Howard and Nassim Rad, "Electrodiagnistic testing for the Diagnosis and management of amyotrophic lateral sclerosis," Physical Medicine \& Rehabilitation Clinics of North America, vol. 29, no. 4, pp. 669-680, 2018.

[8] S. Kian-bostanabad and M. Azghani, "The relationship between RMS electromyography and thickness change in the skeletal muscles," Medical Engineering \& Physics, vol. 43, pp. 92-96, 2017.

[9] A. Abdelouahad, A. Belkhou, A. Jbari, and L. Bellarbi, "Time and frequency parameters of sEMG signal-force relationship," 2018 4th Int. Conference on Optimization and Applications (ICOA), Mohammedia, pp. 1-5, 2018.

[10] Q. Ai, Q. Liu, W. Meng, and S. Q. Xie, "Chapter 3-Neuromuscular signal acquisition and processing," Advanced Rehabilitative Technology: Neural Interfaces and Devices, Academic Press, pp. 33-66, 2018.

[11] A. B. M. S. U. Doulaht and M. A. Jumana, "ALS disease detection in EMG using time - frequency method," 2012 International Conference on Informatics, Electronics \& Vision (ICIEV), Dhaka, pp. 648-651, 2012. 
[12] T. Kamali, R. Boostani, and H. Parsaei, "A multi-classifier approach to MUAP classification for diagnosis of neuromuscular disorders," IEEE Trans. on Neural Syst. and Rehabilitation Eng., vol. 22, no. 1, pp. 191-200, 2014.

[13] E. F. Shair, S. A. Ahmad, M. H. Marhaban, S. B. M. Tamrin, and A. R. Abdullah, "EMG processing based measures of fatigue assessment during manual lifting," Biomed Research Int., vol. 2017, no. 3, pp. 1-12, 2017.

[14] R. H. Chowdhury, M. B. L. Reaz, Mohd A. Mohd Ali, A. A. A. Bakar, K. Chellappan, and T. G. Chang, "Surface electromyography signal processing and classification techniques," Sensors, vol. 13, no. 9, pp. 12431-12466, 2013.

[15] J. Youse and A. Hamilton-wright, "Characterizing EMG data using machine-learning tools," Computers in Biology and Medicine, vol. 51, pp. 1-13, 2014.

[16] N. Nazmi, A. Rahman, Mohd Azizi, S-I. Yamamoto, S. A. Ahmad, H. Zamzuri, and S. A. Mazlan, "A review of classification techniques of EMG signals during isotonic and isometric contractions," Sensors, vol. 16, no. 8, pp. 1-28, 2016.

[17] A. B. M. S. Ud Doulah, S. A. Fattah, W-P. Zhu, and M. O. Ahmad, "Wavelet domain feature extraction scheme based on dominant motor unit action potential of EMG signal for neuromuscular disease classification," IEEE transactions on Biomedical Circuits and Systems, vol. 8, no. 2, pp. 155-164, 2014.

[18] M. Markovska and D. Taskovski, "The effectiveness of wavelet based features on power quality disturbances classification in noisy environment," 2018 18th Int. Conference on Harmonics and Quality of Power, pp. 1-6, 2018.

[19] R. Boostani, F. Karimzadeh, and M. Nami, "A comparative review on sleep stage classification methods in patients and healthy individuals," Computer Methods and Programs in Biomedicine, vol. 140, pp. 77-91, 2017.

[20] M. Li, W. Chen, and T. Zhang, "Classification of epilepsy EEG signals using DWT-based envelope analysis and neural network ensemble," Biomedical Signal Processing and Control, vol. 31, pp. 357-365, 2017.

[21] A. Belkhou, A. Jbari, and L. Belarbi, "A continuous wavelet based technique for the analysis of electromyography signals," 2017 International Conference on Electrical and Information Technologies (ICEIT), Rabat, pp. 1-5, 2017.

[22] D. Chen, S. Wan, J. Xiang, and F. S. Bao, "A high-performance seizure detection algorithm based on Discrete Wavelet Transform (DWT) and EEG," PLoS One, vol. 12, no. 3, pp. 1-21, 2017.

[23] S. G. Mallat, "A theory for multiresolution signal decomposition: the wavelet representation," IEEE Transactions on Pattern Analysis and Machine Intelligence, vol. 11, no. 7, pp. 674-693, 1989.

[24] M. Aqil, A. Jbari, and A. Bourouhou, "ECG signal denoising by discrete wavelet transform," International Journal of Online and Biomedical Engineering, vol. 13, no. 9, pp. 51-68, 2017.

[25] K. Hu, Z. Liu, and S. Lin, "Wavelet entropy-based traction inverter open switch fault diagnosis in high-speed railways," Entropy, vol. 18, no. 3, 2016.

[26] L. Yang, M. D. Judd, and C. J. Bennoch, "Denoising UHF signal for PD detection in transformers based on wavelet technique," The 17th Annual Meeting of the IEEE Lasers and Electro-Optics Society, pp. 166-169, 2004.

[27] A. F. Pérez-Vidal, C. D. Garcia-Beltran, A. Martinez-Sibaja, and R. Posada-Gomez, "Use of the stockwell transform in the detection of P300 evoked potentials with low-cost brain sensors," Sensors, vol. 18, no. 5, pp. 1-14, 2018.

[28] N. R. Babu and B. J. Mohan, "Fault classification in power systems using EMD and SVM," Ain Shams Engineering Journal, vol. 8, no. 2, pp. 103-111, 2017.

[29] R. Sharma, R. B. Pachori, and U. R. Acharya, "Application of entropy measures on intrinsic mode functions for the automated identification of focal electroencephalogram signals," Entropy, vol. 17, no. 2, pp. 669-691, 2015.

[30] X. Chen, X. Zhu, and D. Zhang, "A discriminant bispectrum feature for surface electromyogram signal classification," Medical Engineering \& Physics, vol. 32, no. 2, pp. 126-135, 2010.

[31] R. Bajric, N. Zuber, G. A. Skrimpas, and N. Mijatovic, "Turbine gearbox feature extraction using discrete wavelet transform for gear fault diagnosis of wind turbine gearbox," Shock and Vibration, vol. 2016, no. 1, pp. 1-10, 2016.

[32] L. Zhang, K. Liu, Y. Wang, and Z. B. Omariba, "Ice detection model of wind turbine blades based on random forest classifier," Energies, vol. 11, no. 10, 2018.

[33] K. PU, A. N, T. S, and B. V, "TQWT based features for classification of ALS and healthy EMG signals," American Journal of Computer Science and Information Technology, vol. 6, no. 2, pp. 1-7, 2018.

\section{BIOGRAPHIES OF AUTHORS}

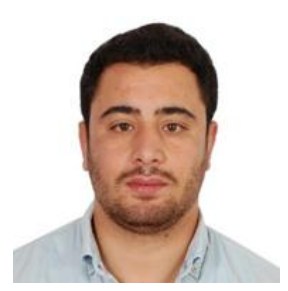

Mr. Abdelouahad Achmamad received his master degree in electrical engineering from high school of technical education, Rabat. Currently $\mathrm{PhD}$ student with research team of electronic systems sensors and Nano biotechnologies (ESSN) at ENSET, Mohammed V University in Rabat, Morocco. He is interested in embedded systems and signal processing.

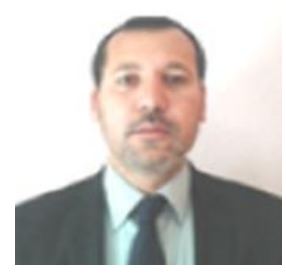

Dr. Atman Jbari is currently a Professor at the electrical engineering department of ENSET "Ecole Normale Supérieure de l'Enseignement Technique", Mohamed V University in Rabat, Morocco. In 2009, he received his PhD in computer and telecommunications from Mohammed V-Agdal university. His current research interests include signal processing, blind source separation and embedded electronic systems. He is member of Electronic Systems, Sensors and Nanobiotechnology research group. 